\title{
Direct observation of spatio-temporal dynamics of short electron bunches in storage rings
}

\author{
C. Evain,,${ }^{1, *}$ E. Roussel, ${ }^{2}$ M. Le Parquier, ${ }^{1}$ C. Szwaj, ${ }^{1}$ M.-A. \\ Tordeux, ${ }^{3}$ L. Manceron, ${ }^{3}$ J.-B. Brubach, ${ }^{3}$ P. Roy, ${ }^{3}$ and S. Bielawski ${ }^{1}$ \\ ${ }^{1}$ Laboratoire de Physique des Lasers, Atomes et Molécules, UMR CNRS 8523 \\ Centre d'Études et de Recherches Lasers et Applications, FR CNRS 2416, Université \\ des Sciences et Technologies de Lille, F-59655 Villeneuve d'Ascq Cedex, France \\ ${ }^{2}$ Elettra-Sincrotrone Trieste, Strada Statale 14-km 163,5 in AREA Science Park,34149 Basovizza, Trieste, Italy \\ ${ }^{3}$ Synchrotron SOLEIL, Saint Aubin, BP 34, 91192 Gif-sur-Yvette, France
}

(Dated: October 17, 2018)

\begin{abstract}
In recent synchrotron radiation facilities, the use of short (picosecond) electron bunches is a powerful method for producing giant pulses of Terahertz Coherent Synchrotron Radiation ( $\mathrm{THz}$ CSR). Here we report on the first direct observation of these pulse shapes with a few picoseconds resolution, and of their dynamics over a long time. We thus confirm in a very direct way the theories predicting an interplay between two physical processes. Below a critical bunch charge, we observe a train of identical THz pulses (a broadband Terahertz comb) stemming from the shortness of the electron bunches. Above this threshold, a large part of the emission is dominated by drifting structures, which appear through spontaneous self-organization. These challenging single-shot $\mathrm{THz}$ recordings are made possible by using a recently developed photonic time stretch detector with a high sensitivity. The experiment has been realized at the SOLEIL storage ring.
\end{abstract}

PACS numbers: 41.60.Ap, 29.27.Bd, 42.65.Re, 07.57.Hm

In last generation storage ring facilities, it has become possible to produce pulses of Coherent Synchrotron Radiation (CSR), with powers that exceed classical (incoherent) synchrotron radiation by factors greater than 10000 [1-7], and may be organized in Terahertz combs [8, 9].

A priviledged way for obtaining this type of emission with the stability required for users, consists in operating the storage ring facilities with short (picosecond) electron bunches $[2,6,7,10-14]$. As the main idea, if the longitudinal shape of an electron bunch presents Fourier components at terahertz frequencies, an intense and stable emission of coherent terahertz radiation is generally expected in bending magnets of accelerators, as illustrated in Figure 1.

This simple conceptual picture is nevertheless deeply complicated by a physical phenomenon of very fundamental nature: the interaction of electron bunches with their own emitted radiation leads to a spatiotemporal instability, which is characterized by the spontaneous appearance of microstructures at the millimeter scale [1517]. In turn, these structures also emit CSR. Hence the interplay between these two modes of emission (i.e., from the "shortness" of the electron bunches and from the spontaneously formed microstructures) plays a central role in the knowledge and improvement of the present and future CSR sources.

However, it is only recently that direct experimental observation of the emitted $\mathrm{THz}$ electric field pulse shapes became possible. As an important milestone, electrooptic sampling has become possible in storage rings ei-

\footnotetext{
*clement.evain@univ-lille1.fr
}

ther for recording CSR pulses [18, 19], and electron bunch near-field shapes [20]. Then the so-called photonic timestretch strategy [21, 22] opened the way to direct recordings of CSR electric field pulse shapes, over successive shots at ultra-high repetition rates [23].

Nevertheless, the limited sensitivity of these new highrepetition-rate picosecond detectors limited experimental investigation of $\mathrm{THz}$ signals to special conditions of high bunch charge and long electron bunches. This configuration corresponds to highest possible peak powers, but also to strongly unstable operation $[17,23]$. Thus, characterization of CSR pulse shape dynamics remained an open question for short (picosecond-scale) and low-charge electron bunches, i.e., the specific conditions where exploiting this fundamental phenomenon, as a $\mathrm{THz}$ source, are foreseen.

In this Letter, we present the actual shapes (the electric field evolution, including envelope and carrier) of the Terahertz CSR pulses emitted in short bunch operation in a storage ring. This analysis has been possible by combining two recent advances in Terahertz electric field detection: high-sensitivity electro-optic sampling [24, 25] for obtaining the required signal-to-noise ratio with picosecond resolution, and the so-called time-stretch strategy [21-23], which enables single-shot recordings at ultra-high acquisition rates. As a main result, we present a direct characterization of the transition from stable Terahertz emission to dynamically evolving pulses: the microbunching instablity threshold, which plays a central role in theories of CSR $[15,16]$. We also show that numerical integration of the Vlasov-Fokker-Planck equation reproduces the main characteristics of the experimental signals. 


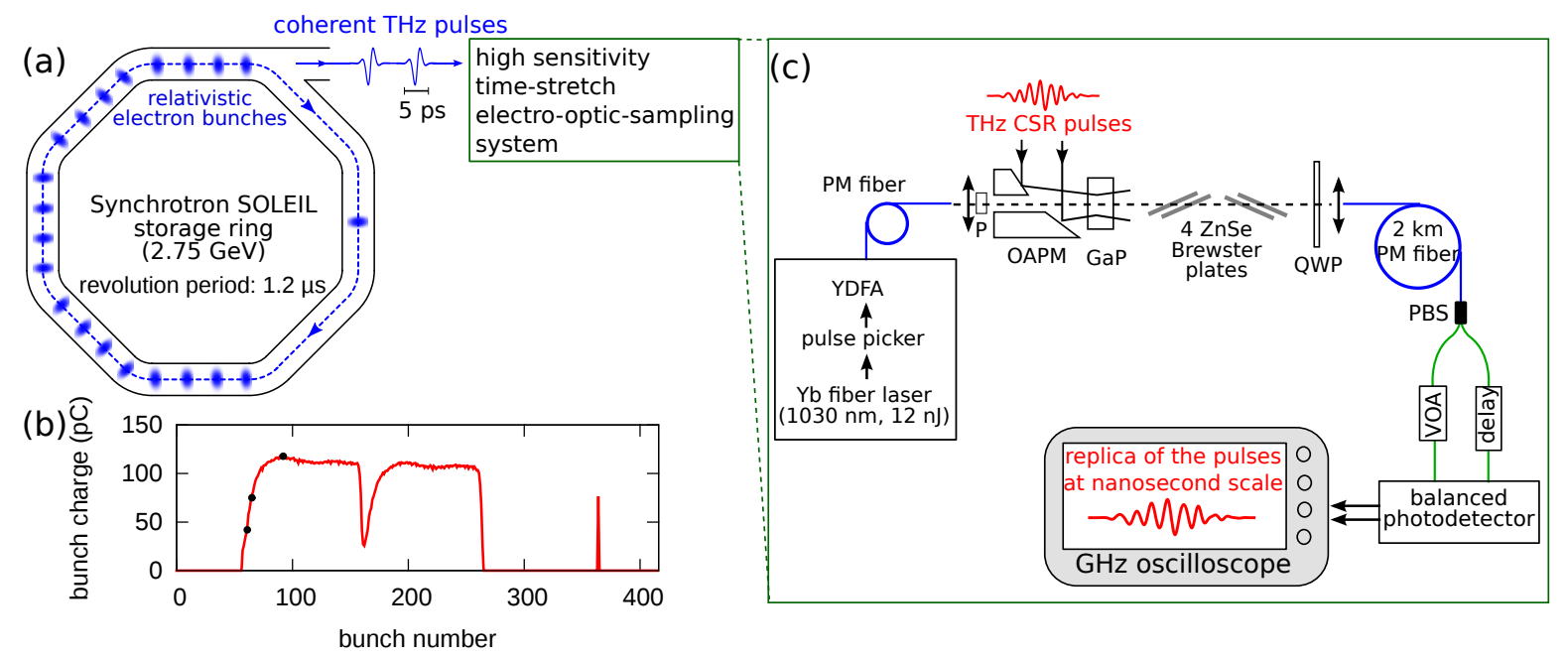

FIG. 1. a) Global experimental arrangement. The SOLEIL storage ring is operated with many short electron bunches (209 in the experiment). The $\mathrm{THz}$ coherent synchrotron radiation pulses (CSR) emitted in a bending magnet are recorded in single-shot with picosecond resolution at the THz/IR AILES beamline, using high-sensitivity photonic time-strech electro-optics sampling. (b) Distribution of the electron bunches charges over one turn for this experiment. The three dots indicate the electron bunches whose CSR pulses evolutions are displayed in Figures 2 and 3. (c) photonic time stetch electro-optical sampling setup. Blue lines: polarization-maintaining (PM) fibers (PM980). green lines lines: single mode fibers (HI1060). PBS: fibered polarizing beam-splitter. OAPM: Off-Axis parabolic mirror, P: polarizer, GaP: $5 \mathrm{~mm}$ long, [110]-cut Gallium Phosphide crystal, QWP: quarter-wave plate, VOA: variable optical attenuator, delay: adjustable delay line. The balanced detector is an DCS-R412 from Discovery Semiconductors. The use of the four ZnSe plates enables to enhance the setup detectivity (see text and Ref. [24]). The polarizations of the laser and $\mathrm{THz}$ field are parallel and oriented along the [-110] axis of the GaP crystal (and peremptory to the Figure plane). The QWP is adjusted at 45 degrees with respect to the laser polarization.

The experiment has been performed at the SOLEIL storage ring in a so-called low-alpha user shift. This mode is characterized by the operation of short bunches (of about 5 ps RMS [26]), which emit CSR with a stability which is compatible with $\mathrm{THz}$ user experiments. Moreover the 209 bunches which circulate in the storage ring have typically different charges [Fig. 1(b)]. This allows investigations as a function of bunch charge by simply studying the emission of each individual electron bunches, without change of the storage ring operating conditions.

The THz CSR pulses are recorded on the AILES beamline, using a photonic time-stretch electro-optic sampling setup, which is specially designed for high sensitivity [Fig. 1(c)]. This detection system is composed of two parts, which are partly intertwined (see also Ref. [27] for details). The first part is a single-shot EOS $[28,29]$ system based on the design developped at SLS [19], FLASH [30] and ANKA [20], which imprints the $\mathrm{THz}$ electric evolution onto the longitudinal profile of a chirped laser pulse at $1030 \mathrm{~nm}$. As a key point for reaching the required high sensitivity, we follow the special strategy recently published by Ahmed, Savolainen and Hamm [24, 25], which consists in using balanced detection, and introducting a set of Brewster plates as shown in Figure 1(c). In order to obtain the necessary high acquisition rates, we associated this EOS setup with the so-called photonic time stretch technique developed by B. Jalali and coworkers $[21,31]$. The chirped laser pulses

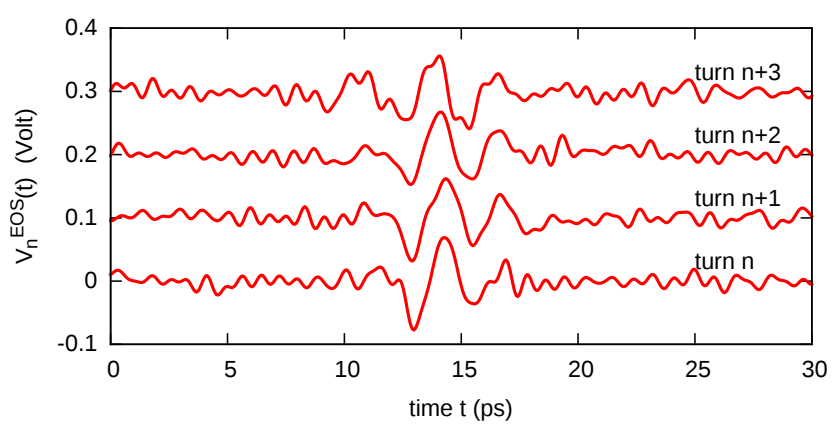

FIG. 2. Typical single-shot electro-optic sampling traces $V_{n}^{E O S}(t)$ (corresponding to the THz CSR electric field emitted by one electron bunch, at four successive turns in the storage ring). The electron charge is $75 \mathrm{pC}$ [second dot, i.e bunch number \#65 on Figure 1(b)]. For clarity, the signals are shifted vertically by $0.1 \mathrm{~V}$.

containing the $\mathrm{THz}$ pulse shape information are stretched within a $2 \mathrm{~km}$-long fiber [22], up to the nanosecond scale. At the balanced photodetectors output, we thus obtain a "replica" of CSR pulse, that is temporally magnified, by a factor of about 200. The optical performances of the setup are described in detail in Ref. [27]. The acquisition window is of the order of $15 \mathrm{ps}$, and the sensitivity is of $2.5 \mu \mathrm{V} / \mathrm{cm} / \sqrt{\mathrm{Hz}}$ at $300 \mathrm{GHz}$ in the electro-optic crystal (i.e., $1.25 \mathrm{~V} / \mathrm{cm}$ ). Since the frequency range of the CSR pulses is typically in the $150-600 \mathrm{GHz}$ range $[6,14]$, the 

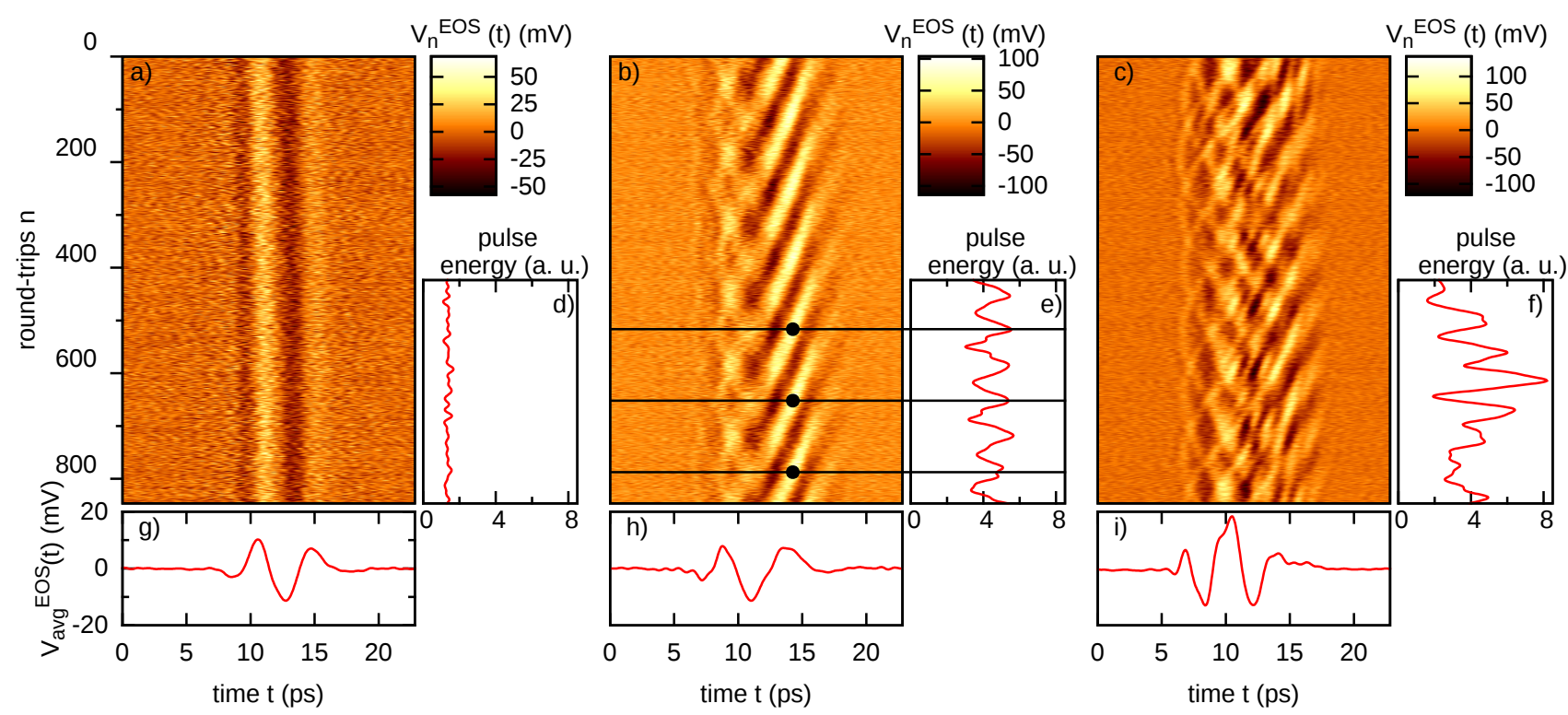

FIG. 3. (a)-(c): Terahertz pulses shapes (electro-optic sampling signal $V_{n}^{E O S}(t)$ ) versus round-trips $n$ in the storage ring. below and above the microbunching instability threshold. (a)-(c) correspond to bunch charges of Q=42 pC, $75 \mathrm{pC}$ and $118 \mathrm{pC}$ respectively [i.e, bunch numbers \#61, \#65, and \#92 in Fig. 1(b)]. Each horizontal cut corresponds to the electric field shape (as in Fig. 2). (d)-(f): pulse energy versus turn number (integration over the time of the square of the EOS signal, and low-pass filtered at $50 \mathrm{kHz})$. Horizontal black lines and dots placed for visual guide. (g)-(i): average electro-optic signal $V_{a v g}^{E O S}(t)$. Note that the data (a) and (b) are recorded simultaneously (i.e., they correspond to a single oscilloscope recording).

stretched signal to be recorded by the oscilloscope is in the $0.75-3 \mathrm{GHz}$ range. Here we use a Teledyne LeCroy LabMaster 10-65Zi oscilloscope (with an - overdimensioned $-30 \mathrm{GHz}$ bandwidth), and the acquired data are numerically low-pass filtered at $6 \mathrm{GHz}$. We recorded 3 ms long time series, which corresponds to approximately 2500 turns in the storage ring. Typical single-shot recordings of the $\mathrm{THz}$ CSR pulses (electric field versus time) are represented in Figure 2. In order to obtain a synthetic view of the CSR pulse dynamics over a large number of turns in the storage ring, we also represented the pulse series in a colorscale diagram, versus time and number of turns, as displayed in Figure 3(a)-(c), for three values of bunch charge.

These diagrams clearly reveal two different emission processes. First, the data evidence the coherent emission which was predicted to occur from the "shortness" of the electron bunch [32]. This process is directly visible below the microbunching instability threshold [Fig. 3(a)]. The emission occurs as a few-period coherent $\mathrm{THz}$ pulses, without dynamical evolution from turn-to-turn. This corresponds to a quasi-perfect Terahertz frequency comb [9].

A second process occurs when the charge exceeds the so-called microbunching instability threshold [Figs. 3(b,c)]. An important part of the $\mathrm{THz}$ radiation is then emitted in the form of an oscillation, that drifts at each turn in the storage ring. This carrier-envelope phase drift stems from the appearance of microstructures which are continuously moving inside the electron bunch, as described in the numerical simulations presented later. In addition, the first process, i.e. the coherent emission due to the bunch shortness, is expected to exist above the microbunching instability threshold. The signature of this contribution from the data presented in Figs. 3(b,c) can be evidenced by computing the electric field pulse average over a large number of turns:

$$
V_{\text {avg }}^{E O S}(t)=\sum_{n=n 0}^{n 0+N-1} V_{n}^{E O S}(t),
$$

where $V_{n}^{E O S}(t)$ is the electro-optic sampling signal of the $\mathrm{THz}$ pulses. This processing filters out the drifting component and may thus be viewed as a measure of the component due to the bunch shortness. This contribution $V_{\text {avg }}^{E O S}(t)$ (with $N \simeq 850$ ) is visible in Figures $3(\mathrm{~g}, \mathrm{~h}, \mathrm{i})$. Note that this component is linked to the short-bunch mode operation, i.e., is not observed when long electron bunches are used [17, 23].

From these data, it is also possible to reconstruct the turn-by-turn evolution of the $\mathrm{THz}$ pulse energy $E_{T H z}^{n}$, with

$$
E_{T H z}^{n}=\int_{t_{1}}^{t_{2}}\left|V_{n}^{E O S}(t)\right|^{2} d t
$$


where $t_{1}$ and $t_{2}$ are taken near the boundaries of the $\mathrm{THz}$ pulse. This signal corresponds to the signal that would be recorded with a standart "slow" detector [see Figs. 3(d-f)]. It permits to confirm in a very direct way that the modulation in the $\mathrm{THz}$ signal, which can be clearly seen in Fig. 3(e) and which had been observed in numerous studies [12, 33-38], is directly related to the period of apparition of the micro-structures in the bunch [Fig. 3(b)].

We have then performed numerical integrations of analytic models, to make a comparison with these experimental data (obtained for the first time in a short-bunch mode), and also to access the 2D longitudinal phasespace. We present here the results obtained with one of the most elementary models: the one-dimensional Vlasov-Fokker-Planck model with shielded CSR wakefield [16]. The evolution of the electron density is described by the phase space distribution $f(q, p, \theta)$, where $\theta$ is the time associated to the number of round-trips in the ring, and $q$ and $p$ are longitudinal coordinate, and electron energy variables respectively. The dynamical evolution of $f(q, p, \theta)$ is determined by the following Vlasov-Fokker-Planck equation [16]:

$$
\frac{\partial f(q, p, \theta)}{\partial \theta}-p \frac{\partial f}{\partial q}+\frac{\partial f}{\partial p}\left(q-I_{c} E_{w f}\right)=2 \epsilon \frac{\partial}{\partial p}\left(p f+\frac{\partial f}{\partial p}\right)(3)
$$

The time $\theta$ associated to round-trips is a dimensionless variable: $\theta=2 \pi f_{s} t$, where $t$ is the time (in seconds) and $f_{s}$ is the synchrotron frequency. The longitudinal position $q$ and relative momentum $p$ are the deviation from the so-called synchronous electron (with position $z_{0}$ and energy $\left.E_{0}\right) . \quad q$ and $p$ are expressed in units of the equilibrium bunch length $\sigma_{z}$ and energy spread $\sigma_{E}$ at zero current: $q=\left(z-z_{0}\right) / \sigma_{z}$, and $p=\left(E-E_{0}\right) / \sigma_{E}$. $\epsilon=1 /\left(2 f_{s} \tau_{s}\right)$, where $\tau_{s}$ is the synchrotron damping time. $I_{c}=I \frac{e 2 \pi R_{c}}{2 \pi f_{s} \sigma_{E} T_{0}}$, with $I$ is the average beam current $\left(I=\frac{Q}{T_{0}}\right), R_{c}$ the dipole radius of curvature, and $T_{0}$ the revolution period. All parameters are in MKS units.

The interaction between electrons is described by the term $E_{w f}(q, \theta)$ which represents the electron bunch wakefield (expressed in $\mathrm{V} / \mathrm{m}$, per Ampere of average current in the storage ring). We use here the CSR wakefield created by an electron bunch in a circular trajectory, between two parallel plates with distance $2 \mathrm{~h}$. The exact expression of $E_{w f}(q, \theta)$ is well known [39], and detailed in [35]. Integration of Eq. (1) is performed using the semi-Lagrangian scheme, described in Ref. [40], and parameters are listed in the figure 4 caption.

Figure 4 shows the results of the numerical integration of equation (1) for three bunch charges $Q=82.6 \mathrm{pC}$, $94.4 \mathrm{pC}$ and $141.6 \mathrm{pC}$. We retreive here qualitatively important features observed experimentally. Below the instability threshold, no micro-structures are observable on the phase-space [Fig. 4(a)] and the shape of the electron bunch is constant from turn-to-turn [Fig. 4(d)]. Just above the instability threshold, "branches" continuously appears on the bottom part of the phase-space, and display a rotational motion at the synchrotron frequency
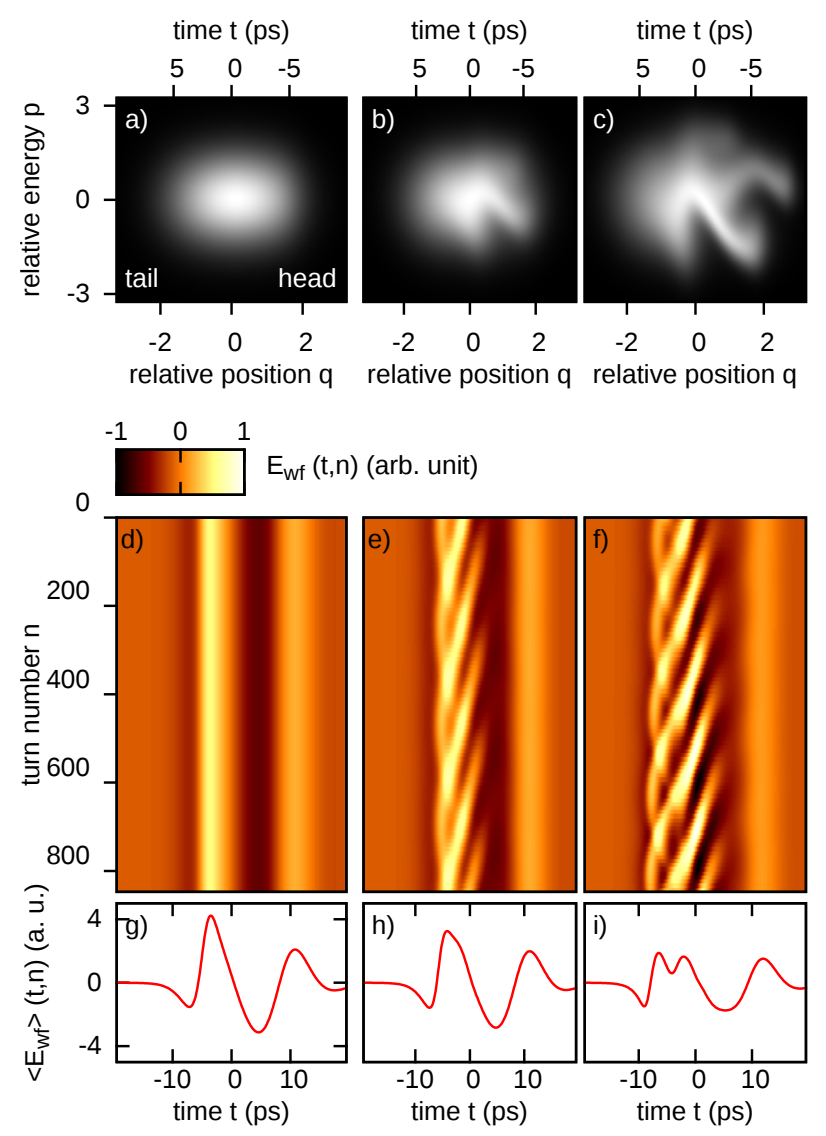

FIG. 4. Numerical simulations of the bunch dynamics, with a charge Q of $82.6 \mathrm{pC}, 94.4 \mathrm{pC}$, and $141.6 \mathrm{pC}$, left, middle and right column respectively. (a)-(c): electron distribution in the phase-space (at the turn number $n \simeq 846$ ). (d)-(f): evolution of the electric field pulses (inside the chamber) $E_{w f}(t, n)$ versus turn number, with $t=-q \sigma_{z} / c, n=\theta /\left(2 \pi f_{s} T_{0}\right), c$ the light velocity. (g)-(i): average over the turns of the emitted pulses. Simulation parameters: $E_{0}=2.75 \mathrm{GeV}, f_{s}=928 \mathrm{~Hz}$, $\sigma_{E}=E_{0} \times 1.017 \times 10^{-3}, \sigma_{z}=0.92 \mathrm{~mm}, \tau_{s}=3.27 \mathrm{~ms}$, $R_{c}=5.36 \mathrm{~m}, T_{0}=1.181 \mu \mathrm{s}, h=12.5 \mathrm{~mm}$.

[see Fig. 4(b) and supplemental movie]. As a main consequence, the wakefield presents an oscillation which is drifting toward the bunch head [Fig. 4(e)]. For higher currents, the micro-structures are more prominent [see Fig. 4(c) and supplemental movie] and can reach the upper part of phase space. In this case, the wakefield [Fig. 4(f)] also presents an oscillation which is drifting toward the tail of the bunch (in addition to the oscillation drifting to the head).

We believe that the availability of this new type of data (CSR pulse shapes versus round-trips) is a precious tool for testing models of bunch dynamics (including wakefields), in a more clear-cut way than with classical means (i.e., average spectra, $\mathrm{THz}$ pulse energies, microbunching instability threshold, etc.). In the example presented here (where one of the most elementary model of wakefields is used), agreement as well as differences can clearly be 
seen. For instance below threshold, the wakefield displays a longer period in the numerical simulation [Fig. 4(g)] than experimentally [Fig. 3(g)]. This may be attributed to differences in the wakefield modeling and/or in filter effects in the beamline wich are not taken into account in the modeling. Differences at higher current are also perceptible, as experimental data [Fig. 3(c)] are more irregular than numerical ones [Fig. 4(f)]. We think that such comparisons of numerical and experimental diagrams can now be used as an important guide for the modeling process, including the crucial work of accelerator impedance modeling.

In conclusion, it is now possible to analyze in a complete way the coherent synchrotron radiation emission due to short electron bunches in storage rings. As a main result, we could directly visualize the predicted transi- tion between a stable $\mathrm{THz}$ coherent emission (due to the "bunch shortness") and the emission due to the so-called microbunching instability. In the future, we believe that this viewing of CSR dynamics enables new and stringent tests for modeling the present and future storage ring facilities. In another perspective, there results show that $\mathrm{THz}$ spectra can be obtained at a $\mathrm{MHz}$ repetition rate, paving the way for ultra high repetition rate time resolved $\mathrm{THz}$ spectroscopy.

We would like to thanks Menlo Systems for important advices. The work has been supported by the BQR of Lille University (2015), by the Ministry of Higher Education and Research, Nord-Pas de Calais Regional Council and European Regional Development Fund (ERDF) through the Contrat de Projets État-Région (CPER) 20072013, and by the LABEX CEMPI project (ANR11-LABX-0007).
[1] G. L. Carr, S. L. Kramer, J. B. Murphy, R. P. S. M. Lobo, and D. B. Tanner, Nucl. Instrum. Methods Phys. Res., Sect. A 463, 387 (2001).

[2] M. Abo-Bakr, J. Feikes, K. Holldack, G. Wüstefeld, and H.-W. Hübers, Phys. Rev. Lett. 88, 254801 (2002).

[3] J. M. Byrd, W. P. Leemans, A. Loftsdottir, et al., Phys. Rev. Lett. 89, 224801 (2002).

[4] A. Mochihashi, M. Hosaka, M. Katoh, M. Shimada, and S. Kimura, EPAC 2006 THPLS042, 3380 (2006).

[5] E. Karantzoulis, G. Penco, A. Perucchi, and S. Lupi, Infrared Physics \& Technology 53, 300 (2010).

[6] J. Barros, C. Evain, L. Manceron, et al., The Review of scientific instruments 84, $033102(\overline{2013)}$.

[7] B. E. Billinghurst, J. C. Bergstrom, C. Baribeau, et al., Phys. Rev. Lett. 114, 204801 (2015).

[8] J. L. Steinmann, M. Brosi, E. Bruendermann, et al., 2015 International Particule Accelerator Conference (2015).

[9] S. Tammaro, O. Pirali, P. Roy, et al., Nature communications 6 (2015).

[10] M. Abo-Bakr, J. Feikes, K. Holldack, et al., Phys. Rev. Lett. 90, 094801 (2003).

[11] Y.-L. Mathis, B. Gasharova, and D. Moss, Journal of Biological Physics 29, 313 (2003).

[12] J. Feikes, M. von Hartrott, M. Ries, et al., Phys. Rev. ST Accel. Beams 14, 030705 (2011).

[13] I. P. S. Martin, G. Rehm, C. Thomas, and R. Bartolini, Phys. Rev. ST Accel. Beams 14, 040705 (2011).

[14] J. Barros, C. Evain, E. Roussel, et al., Journal of Molecular Spectroscopy 315, 3 (2015).

[15] G. Stupakov and S. Heifets, Phys. Rev. ST Accel. Beams 5, 054402 (2002).

[16] M. Venturini and R. Warnock, Phys. Rev. Lett. 89, 224802 (2002).

[17] E. Roussel, C. Evain, C. Szwaj, et al., Phys. Rev. Lett. 113, 094801 (2014).

[18] I. Katayama, H. Shimosato, M. Bito, et al., Applied Physics Letters 100, 111112 (2012).

[19] F. Müller, P. Peier, V. Schlott, et al., Phys. Rev. ST Accel. Beams 15, 070701 (2012).

[20] N. Hiller, A. Borysenko, E. Hertle, et al., Proceedings of
IBIC2014 (2014).

[21] F. Coppinger, A. S. Bhushan, and B. Jalali, IEEE Trans. Microw. Theory Techn. 47, 1309 (1999).

[22] K. Goda and B. Jalali, Nature photonics 7, 102 (2013).

[23] E. Roussel, C. Evain, M. Le Parquier, et al., Scientific Reports 5, 10330 (2015).

[24] S. Ahmed, J. Savolainen, and P. Hamm, Review of Scientific Instruments 85, 013114 (2014).

[25] J. Savolainen, S. Ahmed, and P. Hamm, Proceedings of the National Academy of Sciences 110, 20402 (2013).

[26] M.-A. Tordeux, J. Barros, A. Bence, et al., 2012 International Particule Accelerator Conference , 1608 (2012).

[27] C. Szwaj, C. Evain, M. L. Parquier, et al., arxiv , 1607.07168 (2016).

[28] Z. Jiang and X.-C. Zhang, Applied Physics Letters 72, 1945 (1998).

[29] I. Wilke, A. M. MacLeod, W. A. Gillespie, et al., Phys. Rev. Lett. 88, 124801 (2002).

[30] B. Steffen, V. Arsov, G. Berden, et al., Phys. Rev. ST Accel. Beams 12, 032802 (2009).

[31] J. Wong, H. Q. Lam, R. M. Li, et al., J. Lightwave Technol. 29, 3099 (2011).

[32] F. Sannibale, J. M. Byrd, A. Loftsdóttir, et al., Phys. Rev. Lett. 93, 094801 (2004).

[33] P. Kuske, PAC09 , 4682 (2009).

[34] C. Evain, J. Barros, A. Loulergue, et al., EuroPhysics Letters 98, 40006 (2012).

[35] E. Roussel, C. Evain, C. Szwaj, and S. Bielawski, Phys. Rev. ST Accel. Beams 17 (2014).

[36] V. Judin, N. Hiller, A. Hofmann, et al., IPAC12 , 1623 (2012).

[37] R. Bartolini, G. Cinque, I. Martin, G. Rehm, and C. Thomas, IPAC11, 3050 (2011).

[38] B. E. Billinghurst, J. C. Bergstrom, C. Baribeau, et al., Phys. Rev. Accel. Beams 19, 020704 (2016).

[39] J. B. Murphy, S. Krinsky, and R. L. Gluckstern, Part. Accel. 57, 9 (1997).

[40] R. L. Warnock and J. A. Ellison, SLAC PUB , 8404 (2000). 